\title{
Pan masala advertisements are surrogate for tobacco products
}

\author{
Sushma C, Sharang $\mathbf{C}^{1}$ \\ Indian Cancer Society, Delhi Branch and 'Delhi Public School, New Delhi, India \\ Correspondence to: C Sushma, E-mail: sushmachaudhry@yahoo.com
}

\begin{abstract}
BACKGROUND: Pan masala is a comparatively recent habit in India and is marketed with and without tobacco. Advertisements of tobacco products have been banned in India since 1st May 2004. The advertisements of plain pan masala, which continue in Indian media, have been suspected to be surrogate for tobacco products bearing the same name. The study was carried out to assess whether these advertisements were for the intended product, or for tobacco products with same brand name. MATERIALS AND METHODS: The programme of a popular television Hindi news channel was watched for a 24-h period. Programmes on the same channel and its English counterpart were watched on different days to assess whether the advertisements were repeated. The total duration of telecast of a popular brand of plain pan masala (Pan Parag) was multiplied by the rate charged by the channel to provide the cost of advertisement of this product. The total sale value of the company was multiplied by the proportion of usage of plain pan masala out of gutka plus pan masala habit as observed from a different study, to provide the annual sale value of plain pan masala product under reference. RESULTS: The annual sale value of plain Pan Parag was estimated to be Rs. 67.1 million. The annual cost of the advertisement of the same product on two television channels was estimated at Rs. 244.6 million. CONCLUSION: The advertisements of plain pan masala seen on Indian television are a surrogate for the tobacco products bearing the same name.
\end{abstract}

Key words: Gutka, Pan masala, Surrogate advertisement

\section{Introduction}

Advertising is a well-known method of promotion of a product or idea. Various modalities of advertisements are known. Any place an 'identified' sponsor pays to deliver their message through a medium is considered advertising. ${ }^{[1]}$ Although mouth-to-mouth promotion is also considered advertisement, money also is quite often spent to create the buzz. The companies ultimately aim at creating a brand franchise for their product through advertisements. Various modalities for advertisements are known. Tobacco companies also use various methods of advertising for promotion of their product. Having a favorite cigarette advertisement has been observed to be associated with future smoking or likelihood of trying tobacco, ${ }^{[2]}$ as well as with earlier age at smoking initiation. ${ }^{[3]}$ Direct mechanisms by which tobacco advertising may increase tobacco consumption include (i) encouragement of initiation of tobacco; (ii) increase in daily consumption of tobacco; (iii) retarding cessation efforts; (iv) encouraging former smokers to resume. Three indirect mechanisms of such increase may happen through (i) media dependence on tobacco advertising revenues discouraging full and open discussion of its hazards; (ii) support to institutions may create political support or mute oppositions to tobacco industry's marketing and policy objectives; (iii) ubiquity of advertising may contribute to a social environment in which tobacco is perceived to be socially acceptable. ${ }^{[4]}$ On the other hand, tobacco companies consistently deny the role of advertising in promotion of tobacco use. They claim that the purpose and effect of marketing are merely to provide information and to influence brand selection among current users of tobacco products.

In view of its important role in promotion of tobacco use, many countries have banned advertisements of 
tobacco products. However, tobacco companies often circumvent such efforts through trademark diversification by sponsoring various activities ${ }^{[5]}$ or through surrogate advertisements. The literal meaning of 'Surrogate Advertising' is duplicating the brand image of one product extensively to promote another product of the same brand.

India had been concerned about the promotional effect of tobacco advertisements. Tobacco advertisements had been banned on electronic media and on Government controlled print media since the $1980 \mathrm{~s}^{[6]}$ This was subsequently made stricter through Cable Television Network Act, 1995, to cover direct, as well as indirect advertising of tobacco products. However, tobacco advertisements were permitted in print media till the implementation of the comprehensive legislation on control of tobacco products. ${ }^{[7]}$ Formulation and promulgation of this legislation took a substantial time and the tobacco companies were possibly gearing themselves to circumvent such ban.

Smokeless tobacco use is quite prevalent in India. It was estimated that 96 million out 184 million tobacco users $(52 \%)$ of India consumed tobacco in smokeless form. ${ }^{[8]}$ Use of 'Gutka' and 'Pan Masala with Tobacco' is a common modality of tobacco use especially among the youth. It has been reported through large-scale representative surveys in Uttar Pradesh and Karnataka (2001) that 77.25 and $83.1 \%$ of users of gutka or pan masala-containing tobacco, respectively, from the two states, were below the age of 40 years. ${ }^{[9]}$ Four types of pan masala are available in India market, namely, plain pan masala; sweet pan masala; pan masala containing tobacco; gutka. Gutka has been defined as tobacco along with small quantity of pan masala. Growth of pan masala industry was very slow in the 1970s when only plain and sweet pan masala were introduced. However, this industry saw a sharp growth from 1980s onwards, after the introduction of pan masala, containing tobacco and gutka, especially in small sachets (which increased their transportability and cost of single purchase). Often people consider all pan masala to be same and tobacco to be an integral part of the concoction. The smokeless tobacco industry of India seems to have taken advantage of this perception over the last few years and have initiated advertisements of plain pan masala. Some commonly observed advertisements included, Pan Parag, Goa 1000, Shimla, Rajnigandha, etc. While direct advertisements of all tobacco products vanished after promulgation of the comprehensive legislation for tobacco control from lst May 2004, the advertisements of various pan masala continued, apparently suggesting their nontobacco nature. In fact one such product (Pan Parag) had been advertising about its $0 \%$ tobacco contents. In the recent past, a Khaini product advertised itself as 'Chaini Khaini' in print media and on billboards outside Delhi (billboard advertising of tobacco products has been banned in Delhi since I January 1997), but on television the product was mentioned as 'Chaini Chaini', without even mentioning the nature of the product. ${ }^{[10]}$ In recent times, this television advertisement shows a sachet mentioning it as Chaini Chaini pan masala, but with no major change in the advertisement.

Tobacco control activists have long been criticising these advertisements as indirect tobacco advertisements, but had no means to prove it. This preliminary study was carried out to check whether the advertisements of plain pan masala or some other products with names similar to tobacco products were really the advertisements of the intended products or were surrogate advertisements of their tobacco products.

\section{Methodology}

Advertisements of many plain pan masalas or other products with names similar to tobacco products had been observed on television, as well as in print media. Two major television news channels of India were observed to be showing a large number of advertisements of pan masalas or other products bearing similar names as the gutka/chewing tobacco/bidi products. The programmes of two such news channels (Hindi channel Aaj Tak and English channel Headlines Today) were viewed continuously for $24 \mathrm{~h}$ on two different days. The programme of Hindi news channel was seen on 22 May 2005 (from 8.30 PM of 21 May 2005 to 8.30 PM of 22 May 2005), whereas the programmes of English news channel were seen on 15 June 2005 (from 11.00 PM of 14 June 2005 to 11.00 PM of 15 June 2005). The frequency and duration of all these advertisements was noted. The programmes were noted on several other days also to assess whether the frequency of these advertisements was similar.

Other variables important to the study related to the total sale value of the gutka products, and the proportion of sale of plain pan masala to gutka or to the total tobacco products. The tobacco companies may not readily provide information on these aspects. The sale value of tobacco products of one of these companies (M/s Kothari Brothers, manufacturers of Pan Parag pan masala and Pan Parag gutka) was available on the Internet, and this information for the most recent available financial year (April 2003 to March 2004) was used for this study. 
A large sentinel survey on tobacco use pattern was carried out in the year 2001 in the states of Uttar Pradesh and Karnatka. ${ }^{[1]}$ The lead author of that study provided additional information on the prevalence of use of plain pan masala and gutka by these communities as observed in the survey. This data helped in assessing the proportion of users of plain pan masala as compared to users of all types of pan masala (irrespective of presence of tobacco).

The rate of advertising for the television channels were noted from the website of the television channels. There were differential rates for different timings. In view of the high level of advertisement provided by the specific product (Pan Parag) and its 24-h distribution on Hindi news channel, the lowest rate given for bulk advertisers was used for further calculations (Rs. 5500 per $10 \mathrm{~s}$ ). However, the advertisements on the English news channel were concentrated during daytime. Thus, the rate for each of the advertisement was considered, as given by the channel for maximum volume users.

The study adopted the principle of providing the margin of doubt in favor of tobacco companies in case of limited information on the subject. For example, prevalence of consumption of plain pan masala was observed to be more in the state of Uttar Pradesh (as compared to Karnataka). The prevalence of such products was more in urban areas. Therefore, such proportion as observed from data pertaining to urban Uttar Pradesh was used for the study. Similarly, the rates for advertisement, which might have been paid to the television channel were assessed at the minimum chargeable rates by the channel.

\section{Observations}

Advertisements of these products are not limited to the two channels observed under the study. The same advertisements have also been observed on many other television channels. Advertisements of these products also appear in the print media. As such, this study

\begin{tabular}{lll}
\hline \multicolumn{3}{l}{ Table 1: } \\
\multicolumn{2}{l}{ advertisements on two television channels } \\
\hline S. No. & Advertised product & $\begin{array}{l}\text { Name of the product } \\
\text { containing tobacco }\end{array}$ \\
\hline 1 & Pan Parag pan masala & Pan Parag gutka \\
\hline 2 & Rajnigandha pan masala & Rajnigandha tobacco mix \\
\hline 3 & Chaini Chaini pan masala & Chaini Khaini \\
\hline 4 & Gopal Supari 132 & Gopal Zarda \\
\hline 5 & Pataka 502 Tea & Pataka 502 Bidi \\
\hline
\end{tabular}

should only be considered as preliminary, and as estimating only the minimum expenses on advertisements by the concerned companies.

During the 24-h telecast on the selected television news channel, five types of advertisements were observed which had names similar to tobacco products [Table 1]. Three types of advertisements were observed during the telecast of the Hindi news channel. These included, direct advertisements of the product, sponsorship of a programme, and advertisements of a programme on the same channel partially sponsored by these companies. Under the last category, besides mentioning their role as sponsors, details about the product were also mentioned. Total duration of advertisements of these five products turned out to be $1215 \mathrm{~s}$ during the $24 \mathrm{~h}$ under observation with Pan Parag being the highest contributor for $615 \mathrm{~s}$ [Table 2].

Only direct advertisements were observed on the English news channel. These advertisements were not seen after midnight till the early hours of the day. The total duration of these advertisements was $825 \mathrm{~s}$, with Pan Parag contributing $420 \mathrm{~s}$ [Table 3].

The total sale value of one of the product being advertised during this period was available on the Internet. The total sale value of $\mathrm{M} / \mathrm{s}$ Kothari Brothers was Rs. 1676.4 million during the financial year 20032004; Rs. 2315.00 million during the financial year 2002-2003; Rs. 2821.60 million during the financial

\section{Table 2: Duration of advertisements of surrogate tobacco products during 24-h of observation on Hindi news channel Aaj Tak}

\begin{tabular}{|c|c|c|c|c|}
\hline Product & Direct advertisement & Sponsorship of a programme & $\begin{array}{l}\text { Advertisement of a } \\
\text { sponsored programme }\end{array}$ & Total duration \\
\hline Pan Parag pan masala & 290 & 195 & 130 & 615 \\
\hline Pataka 502 Tea & 150 & 70 & 125 & 345 \\
\hline Rajnigandha pan masala & 90 & - & 10 & 100 \\
\hline Chaini Chaini pan masala & 55 & - & - & 55 \\
\hline Gopal Supari 132 & 90 & - & - & 90 \\
\hline All above products & 685 & 265 & 265 & 1215 \\
\hline
\end{tabular}


year 2001-2002; Rs. 2114.80 million during the financial year 2000-2001. The latest available figures were used for the purpose of calculations under the study.

The prevalence of use of plain pan masala, gutka and proportion of plain pan masala to these products is given in Table 4 . In view of the principle of providing the margin of doubt in favor of tobacco companies in case of limited or varied information, the maximum observed proportion of use of plain pan masala (among urban men in Uttar Pradesh at 3.8\%) was used for further calculations in the study.

Using the values noted from various sources, Table 5 shows that the annual sale value of Plain Pan Parag was assessed at Rs. 6.37 crores (Rs. 63.7 million or US \$ 1.45 million at currently prevailing exchange rate). On the other hand, the annual cost of advertisements of the same product on two television channels (Aaj Tak and Headlines Today) was estimated to be Rs. 24.46 crore (Rs. 244.6 million or US \$ 5.56 million at currently prevailing exchange rate).

\section{Discussion}

The annual cost of advertisement of one of the most popular brand of plain pan masala (Pan Parag) far exceeds its annual sale value. The annual cost of advertisement of plain Pan Parag on two television channels alone is 3.8 times its annual sale value. This indicates that the Pan Parag pan masala advertisements are a surrogate for the tobacco product (gutka) the company manufactures under the same brand name. The current estimate of the annual cost of advertisement pertains to only two television channels. It is important to note that similar advertisements have been observed on other television channels, as well as print media. In view of this, the estimated annual advertising cost is an underestimate, and the real differential is likely to be far more than what has been depicted in this study.

The study was carried out with the hypothesis that the advertisements were aimed for promotion of the product itself. Because of this reason any margin of doubt was in favor of the manufacturer of the product.

\section{Table 3: Duration of advertisements of surrogate tobacco products during 24-h of observation on English news channel Headlines Today}

\begin{tabular}{lccc}
\hline Product & Direct advertisement & Sponsorship of a programme & Advertisement of a sponsored programme \\
\hline Pan Parag pan masala & 420 & - & - \\
\hline Pataka 502 Tea & 210 & - & - \\
\hline Rajnigandha pan masala & 100 & - & - \\
\hline Chaini Chaini pan masala & 25 & - & - \\
\hline Gopal Supari 132 & 70 & - & - \\
\hline All above Products & 825 & - \\
\hline
\end{tabular}

Table 4: Prevalence of use of plain pan masala and gutka in Uttar Pradesh (2001)

\begin{tabular}{|c|c|c|c|c|}
\hline & \multicolumn{2}{|c|}{ Urban } & \multicolumn{2}{|c|}{ Rural } \\
\hline & Male (\%) & Female (\%) & Male (\%) & Female (\%) \\
\hline Prevalence of gutka/pan masala with tobacco & 10.2 & 1.4 & 7.8 & 0.8 \\
\hline Prevalence of plain pan masala & 0.4 & - & 0.1 & - \\
\hline Proportion of plain pan masala to total & 3.8 & - & 1.3 & - \\
\hline
\end{tabular}

Courtesy Dr. Kishore Chaudhry, ICMR, based on the ICMR-WHO, SEAR Sentinel study on tobacco use in Uttar Pradesh, India, 2001.

Table 5: Annual sale value and partial cost of advertisement of plain Pan Parag

\begin{tabular}{ll} 
Total sale value (2003-2004) of products of M/s Kothari Brothers & Rs. 167.64 crore \\
\hline Proportion of plain pan masala of M/s Kothari Brothers to total & $3.8 \%$ \\
\hline Annual sale value of plain Pan Parag & Rs. 6.37 crore \\
\hline Annual cost of advertisement of Pan Parag on Hindi news television channel Aaj Tak & Rs. 12.35 crore \\
\hline Annual cost of advertisement of Pan Parag on English news television channel Headlines Today & Rs. 12.11 crore \\
\hline Total annual cost of advertisement of Pan Parag on two news television channels & Rs. 24.46 crore \\
\hline
\end{tabular}

Note: 1 crore $=10$ million. 
The proportion of use of plain pan masala out of total gutka/pan masala use, as observed among men from urban Uttar Pradesh was used, providing the highest possible proportion for sale of plain pan masala. It is likely that if overall proportion of use of plain pan masala (or plain Pan Parag) out of total gutka and pan masala use were available, the proportion (and thus the sale value) would have been much lower. The exact rates paid by the pan masala companies to the television channel are not known to the authors. The estimates used for the Hindi news television channel were the lowest rates shown on its website, although sponsored and specific time advertisements are known to have differential rates.

Although 24-h quantitative observation for these advertisements was for 1 day for each of the channel, these advertisements have been observed for a very long period. Many programmes on the two channels were watched again on several days at different timings to ensure that the observations were not a one-time phenomenon. In fact, the timings of these advertisements were more or less constant every day.

Such calculations could have been carried out for each of the brand under study, but the study was limited to one brand in view of the nonavailability of sale figures of other brands to the authors. However, the highvolume of advertisements and the knowledge that the proportion of people using plain pan masala is small, suggests that these are also likely to be surrogate for their tobacco products.

One could argue that the proportion of sale of this particular brand of plain pan masala may be very high as compared to other brands of plain pan masala. This however, does not seem to be tenable, because most of the popular brands seem to be advertising heavily. Advertisements of other brands of plain pan masala like Shimla, Goa 1000, have also been seen on television, although this study did not find these advertisements on the specific day.

The above makes it clear that the advertisements of plain pan masala are a surrogate for promotion of gutka bearing same brand name. The manufacturers have taken advantage of common community thinking that pan masala always has tobacco in it. In fact this kind of confusion had been seen in scientific literature also wherein studies carried out on mutagenesis by pan masala did not mention if the product contained tobacco or not. These advertisements seem to have increased during the recent years when the possibility of their ban started becoming a reality.
Trademark diversification and surrogate advertisements are known by cigarette manufacturers. ${ }^{[5]}$ Surrogate advertisements by gutka manufacturers in India has been suspected, but could not be proved. Although preliminary, the current paper provides first objective evidence of surrogate advertisement of any tobacco product.

\section{Conclusion}

The advertisements of plain pan masala seen on Indian television are a surrogate for the tobacco products bearing the same name.

\section{Acknowledgments}

The authors are thankful to Dr. Kishore Chaudhry, Deputy Director General (Sr. Grade), Indian Council of Medical Research, New Delhi, for his guidance and for providing important data related to proportion of persons using plain pan masala in the community.

\section{References}

1. http://encyclopedia.laborlawtalk.com/Advertisement.

2. Pierce JP, Choi WS, Gilpin EA, Farkas AJ, Berry CC. Tobacco industry promotion of cigarettes and adolescent smoking. Journal of the American Medical Association 1998;279:511-5.

3. Unger JB, Chen X. The role of social networks and media receptivity in predicting age of smoking initiation: A proportional hazards model of risk and protective factors. Addictive Behaviors 1999;24:371-81.

4. USDHHS. Reducing the health consequences of smoking - 25 years of progress: A report of the Surgeon General. US Department of Health and Human Services, Maryland;1989:500-2.

5. Assunta M and Chapman S. The tobacco industry's accounts of refining indirect tobacco advertising in Malaysia. Tobacco Control 2004; 13:63-70.

6. Chaudhry K, Prabhakar AK, Luthra UK. Tobacco Control in India Search for Strategies. Tobacco \& Health 1990 - The Global War. Proceedings of the seventh world conference on tobacco \& health. Health Department of Western Australia, Perth 1990;3636.

7. GOI. The Gazette of India. Extraordinary, Part II - Section 1, Government of India. New Delhi, Monday, May 19,2003.

8. Chaudhry K. Tobacco control in India. In: Fifty years of cancer control in India. Agarwal SP, Rao YN, Gupta S, Editors. Directorate General of Health Services, Govt. of India, New Delhi, India; 2002. p.

9. Chaudhry K. Prevalence of Use of Pan Masala-containing Tobacco / Gutka in Karnataka and Uttar Pradesh (India). Poster presented in $12^{\text {th }}$ World Conference on Tobacco or Health, Helsinki, 3-8the August 2003.

10. Chaudhry S. Role of Non Governmental Organizations in Implementation of Tobacco Control Legislation in Delhi. Presentation at $12^{\text {th }}$ World Conference on Tobacco or Health, Helsinki, 3-8the August 2003.

11. Chaudhry K, Prabhakar AK. http://w3.whosea.org/EN/Section 1174/section 1462/pdfs/surv/Sentinellndia2001.pdf) 